\title{
Youla-Kucera Based Lateral Controller for Autonomous Vehicle
}

\author{
I. Mahtout ${ }^{1,2}$, F. Navas ${ }^{3}$, D. Gonzalez ${ }^{3}$, V. Milanés ${ }^{1}$ and F. Nashashibi ${ }^{2}$
}

\begin{abstract}
This paper presents the design and implementation of a novel lateral control approach. The proposed control strategy is based on Youla-Kucera parametrization to switch gradually between controllers that are designed separately for big and small lateral errors. The present approach studies the critical problem of initial lateral error in line following. It ensures smooth and stable transitions between controllers and provides a smooth vehicle response regardless of the lateral error. For an initial validation the present work was tested in simulation, implementing a dynamic bicycle model. It has also been tested in real platforms implementing an electric Renault ZOE, with good results when activating the system at different lateral errors. autonomous vehicles Switched controllers parametrization
\end{abstract}

\section{INTRODUCTION}

Automated vehicles are getting more and more attention because of their potential to improve drivers' life, insuring road safety, increasing highway capacity or reducing carbon emission. When it comes to conceive autonomous vehicles, assuring its stability plays a key role. The vehicle motion stability is assured by a control process that is capable to interpret high level decision (i.e. follow a line, avoid an obstacle) into actions according to a given trajectory. Vehicle control can be divided in longitudinal (i.e. the ability to regulate vehicle speed by controlling brake and throttle actuators) and lateral (i.e. vehicle steering regulation to track and follow a reference path) controllers. This paper is focused on the latter one.

Steering control has been widely studied in the last three decades. The first demonstration was carried out in "No Hands Across America" event in mid 90s. Carnegie Mellon University's researchers [6] presented Navlab car that completed around $5000 \mathrm{~km}$. The steering wheel was controlled by correcting lateral offset and heading error. Curvature was used as feedforward in the control loop. The vehicle was semi-autonomous because the throttle and brake pedals were controlled by the driver. In 1998 Prof.Broggi et al [7] demonstrated the ARGO vehicle, that conducted itself along the Italian highway network. The steering system was based on a proportional controller to correct the vehicle deviation with respect to the center lane. In 2005, Stanley vehicle won the second DARPA (Defense Advanced Research Projects

${ }^{1}$ I. Mahtout and V. Milanés are with the Research Department, Renault SAS, 1 Avenue de Golf, 78280 Guyancourt, France imane.mahtout, vicente.milanes arenault.com

${ }^{2}$ F. Nashashibi is with the Robotics and Intelligent Transportation Systems (RITS) team, Inria Paris, 56 rue du Charolais, 75012 Paris, France fawzi.nashashibi @inria.fr

${ }^{3} \mathrm{~F}$. Navas and D. Gonzalez are with the Intelligent Systems Departement, AKKA Technologies, 1 rue arnold schoenberg, 78280 Guyancourt, France. francisco.navas-matos, david-enrique.gonzalez-bautista dakka.eu
Agency) Grand Challenge [8]. Its steering controller was based on a nonlinear feedback of the cross-track error by using estimated velocity, UKF pose and measured steering wheel angle. This approach works well for lower speed, but it neglected the steering column dynamics. In 2012, BRAiVE vehicle was demonstrated in the Intercontinental Autonomous Challenge, developed by Prof. Broggi's team. Its lateral controller included two levels: first, setting a target point in the desired trajectory, at a look ahead distance that vary with respect to the vehicle speed, then converting the curvature in the control point to steering value, by inverting the vehicle dynamic model. To avoid jerky performance, variation in curvature lower than $\pm 5 \%$ was not considered. The vehicle was able to drive in urban areas (i.e. roundabouts). In the same year, the university team of Prof. Sunwoo won the autonomous car competition organized by Hyundai Motor in Korea [10]. The basic idea of the lateral controller is that the vehicle always moves along Ackerman geometry, except for straight movement. The vehicle showed good performance in mixed circuits. In 2013, Mercedes cooperated with different research labs to make an autonomous journey in memorial Berta Benz road. The lateral controller included two parts: first, a feedforward part that calculates the desired yaw rate using trajectory curvature at a target point. This one is set at a look-ahead distance depending on the vehicle speed, in order to compensate the steering column dynamics; second, a feedback part, that minimizes the lateral displacement in order to stabilize the lateral position. Finally, the required steering wheel angle is computed from the desired yaw rate using an inverse stationary single track model. For steady state accuracy, a steering angle offset observer is used to compensate both deviation in the inverse single track model as well as a steering angle offset in the steering actuator [11]. The above described systems are part of automated steering wheel demonstration well known in the literature. However, other implementations have been proposed. In [12] a nested PID controller is used to control the vehicle steering angle. In [13] an $H_{\infty}$ approach is used. A sliding mode controller is validated by experimental results in [14]. In [15] human steering skills are used to emulate manual driving. Lateral controller is based on fuzzy logic approach fed by informations about the car state while human is driving, via genetic algorithms. In [4], inspired by drivers mechanism, authors developed a T\&C controller (Target and Control Driver Steering Model). The generated steering rate is simply the target angle error (angle between the vehicle heading and the target point set at a look-ahead distance) scheduled by a gain feedback, it has been successfully implemented on a bus revenue service as a simple linear control loop. In [5] 
the same approach has been validated on experimental car. The review of the different techniques shows two different approaches when dealing with steering control: 1) the use of complex vehicle model that includes all dynamics, and design suitable control structure that handles nonlinear behaviors and model parameter variations. In this case, the computation time becomes large for real time operation [18]; and 2) the use of linear controller based on a simplified vehicle model, and tuned for a defined use case (i.e. lane keeping, lane changing), which can't satisfy steering performance in different driving situations.

Automated steering systems should be able to address any circumstances ranging from starting the vehicle fully stopped to activating the function with a significant error with respect to the reference trajectory or performing lane changes. For covering all this situations, a single controller will not satisfy all conditions.

This paper proposes a control structure that handles any significant lateral deviation. This deviation can be caused either by a first system activation, avoidance maneuver or even a localization discontinuity. The proposed control structure can cover the above by switching gradually between two different controllers: a lane-tracker controller for one case and a lane change controller for situations with significant lateral errors. Both controllers are implemented in a reconfigurable control structure, based on Youla-kucera parametrization. This control parametrization ensures stability while switching between controllers. In [1][2][3] authors used YoulaKucera parametrized structure in different applications, and presenting encouraging simulation and experimental results. Both controllers are based on $\mathrm{T} \& \mathrm{C}$ approach, for the fact that it's a linear SISO controller already validated by experimental tests.

This paper is organized as follows. Sections II introduces the Youla-Kucera controller structure and its basis mathematical developments. In Section III, the vehicle model is presented with the control strategy and the stability analysis. Section IV presents the simulation results. Section $\mathrm{V}$ presents experimental platform and results. Some concluding remarks and future work directions are given in the end .

\section{YOULA-KUCERA CONTROLLER CONFIGURATION}

This section presents the basis on the proposed technique for controller switching. It relies on Youla-Kucera parametrization of all stabilizing controllers for a given plant (see [1],[2],[15] and [16]). The Youla-Kucera parametrization is based on the doubly coprime factorization.

Let's consider a plant $G \in \mathbb{R}_{m * n}$, mapping $n$ inputs to $m$ outputs.

$$
y=G u, y \in \mathbb{R}_{m} \text { and } u \in \mathbb{R}_{n} .
$$

Different controllers $K_{i} \in \mathbb{R}_{n * m}$ can be designed to stabilize $G$. Stable switching between those controllers is possible using Youla-Kucera parametrization, the plant $G$ and controllers $K_{i}$ need to be factorized to apply the parametrization.

\section{A. Doubly Coprime Factorization}

The plant model and controllers matrix transfer functions are factorized to a product of a stable transfer function matrix and a transfer function matrix with a stable inverse as follows:

$$
\begin{array}{cc}
G=N M^{-1}=\tilde{M}^{-1} \tilde{N} & N, M, \tilde{N}, \tilde{M} \in \mathbb{R} H_{\infty} \\
K_{i}=U_{i} V_{i}^{-1}=\tilde{V}_{i}^{-1} \tilde{U}_{i} & U_{i}, V_{i}, \tilde{U}_{i}, \tilde{V}_{i} \in \mathbb{R} H_{\infty}
\end{array}
$$

This factorization is named coprime when the two transfer function matrix have no commun zeros in $|z|>1$ or $\mathfrak{R}(s)>0$ in continuous case. Knowing that $K_{i}$ stabilizes $G$, the coprime factors must satisfy the following Bezout equations:

$$
\begin{gathered}
\tilde{V}_{i} M-\tilde{U}_{i} N=I, \\
\tilde{M} V_{i}-\tilde{N} U_{i}=I,
\end{gathered}
$$

Or equivalently, and considering (1) and (2) the double Bezout identity :

$$
\left[\begin{array}{cc}
\tilde{V}_{i} & -\tilde{U}_{i} \\
-\tilde{N} & -\tilde{M}
\end{array}\right]\left[\begin{array}{ll}
M & U_{i} \\
N & V_{i}
\end{array}\right]=\left[\begin{array}{cc}
M & U_{i} \\
N & V_{i}
\end{array}\right]\left[\begin{array}{cc}
\tilde{V}_{i} & -\tilde{U}_{i} \\
-\tilde{N} & -\tilde{M}
\end{array}\right]=\left[\begin{array}{ll}
I & 0 \\
0 & I
\end{array}\right]
$$

To calculate those factors [1], we consider a state space descriptions of $G$ and $K_{i}$ :

$$
G:\left[\begin{array}{c|c}
A & B \\
\hline C & D
\end{array}\right] \quad K_{i}:\left[\begin{array}{c|c}
A_{i} & B_{i} \\
\hline C_{i} & D_{i}
\end{array}\right]
$$

Under the controllability assumption of the pairs $(A, B)$ and $\left(A_{i}, B_{i}\right)$, constant stabilizing state space feedback matrix respectively $F$ and $F_{i}$ can be constructed, in that $(A+B F)$ and $\left(A_{i}+B_{i} F_{i}\right)$ have all eigenvalues within the unity circle. State space descriptions of stable coprime factors for $G$ and $K_{i}$ are given as follows : (further details are in [1])

$$
\begin{aligned}
& {\left[\begin{array}{cc}
M & U_{i} \\
N & V_{i}
\end{array}\right]=\left[\begin{array}{cc|cc}
A+B F & 0 & B & 0 \\
0 & A_{i}+B_{i} F_{i} & 0 & B_{i} \\
\hline F & C_{i}+D_{i} F_{i} & I & D_{i} \\
C+D F & F_{i} & D & I
\end{array}\right]} \\
& {\left[\begin{array}{cc}
\tilde{V}_{i} & -\tilde{U}_{i} \\
-\tilde{N} & \tilde{M}
\end{array}\right]=} \\
& {\left[\begin{array}{cc|cc}
A+B Y D_{i} C & B Y C_{i} & -B Y & B Y D_{i} \\
B_{i} Z C & A_{i}+B_{i} Z D C_{i} & -B_{i} Z D & B_{i} Z \\
\hline F-Y D_{i} C & -Y C_{i} & Y & -Y D_{i} \\
Z C & -\left(F_{i}-Z D C_{i}\right) & Z D & Z
\end{array}\right]}
\end{aligned}
$$

where $Y=\left(I-D_{i} D\right)^{-1}$ and $Z=\left(I-D D_{i}\right)^{-1}$. Then coprime factors can be calculated, using the standard transformation from state space description to transfer function matrix.

\section{B. Youla-Kucera Parametrization}

According to Youla-Kucera, all stabilizing controllers for a given plant $\mathrm{G}$ can be written as a function of a stable filter $Q_{i}$ (see [1]):

$$
\begin{aligned}
K_{i}=K\left(Q_{i}\right) & =\left(U_{i-1}+M_{i-1} Q_{i}\right)\left(V_{i-1}+N_{i-1} Q_{i}\right)^{-1} \\
& =\left(\tilde{U}_{i-1}+Q_{i} \tilde{M}_{i-1}\right)\left(\tilde{V}_{i-1}+Q_{i} \tilde{N}_{i-1}\right)^{-1}
\end{aligned}
$$


where, $Q_{i}$ is a stable transfer function with appropriate inputoutput dimensions, called Youla-Kucera parameter.

Thanks to this parametrization, two controllers are involved in the same control structure as shown in Fig.1. For each stable matrix $Q_{i}$, controller reconfiguration from $K_{i-1}$ to $K_{i}$ is carried out by setting $K_{i}=K\left(Q_{i}\right)$. The stability of the closed loop is guaranteed by the stability of $Q_{i}$ and to perform a smooth transition, a scalar factor $\gamma \in[0,1]$ is added to scale $Q_{i}$. When $\gamma=0$ the control signal is completely generated by $K_{i-1} .0<\gamma<1$ generates a control signal combination of $K_{i-1}$ and $K_{i}$ responses. When $\gamma=1$ the control signal is completely generated by $K_{i}$, more details and proof are in[1].

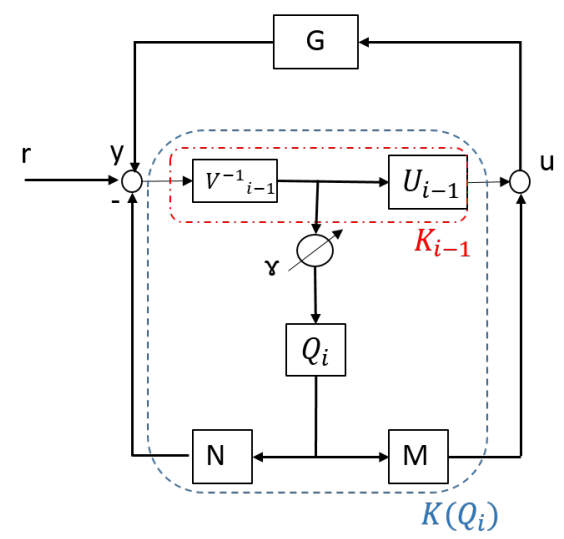

Fig. 1. Youla-Kucera control structure

The steering controller switching proposed in this paper uses the lateral error as parameter to deal with $\gamma$, playing a key role in the final control applied. The design of $\gamma$ is detailed further.

Using two stable controllers $K_{1}$ and $K_{2}$, and including $\gamma$, the parametrized controller is realized as follows:(see [3])

$$
K=\left(U_{1}+M_{1} \gamma Q\right)\left(V_{1}+N_{1} \gamma Q\right)^{-1}
$$

with:

$$
Q=\tilde{U}_{2} V_{1}-\tilde{V}_{2} U_{1}
$$

Other control structures using Youla-Kucera parametrization can be deducted (see [3] for further details).

\section{CONTROLler Design}

The goal of the controller is to develop a system able to deal with not only trajectory following but also with any other circumstance. When passing from manual to autonomous driving (i.e. autonomous driving activation), the vehicle may have an initial error in function of vehicle position with respect to the reference trajectory. The ability to handle this kind of situation can be extended to lane-change maneuver or avoidance maneuver, meaning that it will be hard to handle all circumstances with a single controller. To perform automated lane changing and lane tracking maneuvers, two different steering controller behaviors are required.
Figure 2 shows step response of the expected controller for each situation. In lane-tracking task (solid blue line), the controller must provide fast response to track changes in the desired trajectory (i.e. curved line). Overshoots are tolerated because its maximum value is equal to the initial lateral error, which is small in this case (the vehicle is already in the lane). While in lane-changing case (solid red line), the initial lateral error is significant. Thus, a smooth response is required, less overshoots are provided while compromising the rising time.

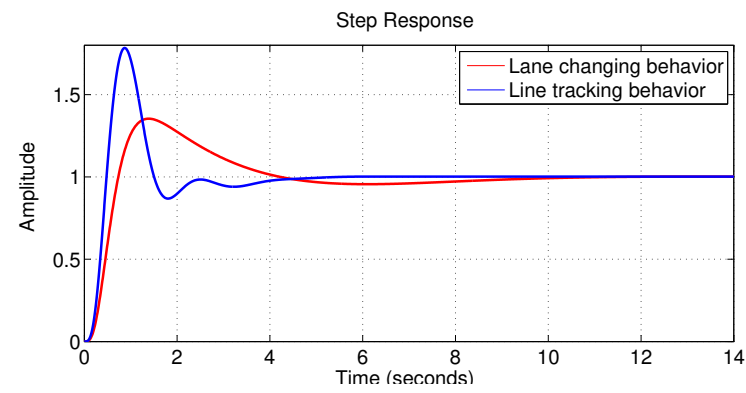

Fig. 2. Desired control behavior

To cover both situations in one control structure, the proposed solution is to design a switched controller. This one is based on Youla-Kucera parametrization to guarantee stable switching (see section II).

The control structure is illustrated in Fig. 3. It includes two controllers: lane-changing controller $C_{1}$ (dashed red box); lane-tracking controller $C_{2}$ (dashed blue box). The switching between controllers is handled by the lateral error between the vehicle and the desired trajectory. Further details on controller design and vehicle model are given below.

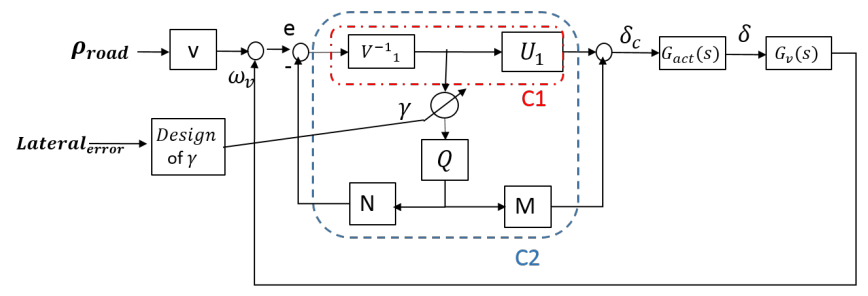

Fig. 3. Control structure

\section{A. Vehicle model:}

The vehicle lateral dynamics is described in a state-space form, using the bicycle model [19] as follows:

$$
\begin{aligned}
\dot{X}_{v} & =A_{v} X_{v}+B_{v} u_{v} \\
Y_{v} & =C_{v} X_{v}
\end{aligned}
$$

where the state vector is:

$$
X_{v}=\left[\begin{array}{llll}
y_{v} & v_{y} & \psi_{v} & \omega_{v}
\end{array}\right]
$$

$y_{v}, v_{y}, \psi_{v}, \omega_{v}$ are the vehicle lateral position, lateral velocity, yaw angle and yaw rate respectively, $u_{v}=\delta_{f} t$ is the steering 
angle in the front tire. The system matrix $A_{v}, B_{v}$ and $C_{v}$ are described below:

$$
\begin{gathered}
A_{v}=\left[\begin{array}{cccc}
0 & 1 & v_{x} & 0 \\
0 & \frac{-\left(C_{f}+C_{r}\right)}{m v_{x}} & 0 & \frac{-a C_{f}+b C_{r}}{m v_{x}}-v_{x} \\
0 & 0 & 0 & 1 \\
0 & \frac{-a^{2} C_{f}+b^{2} C_{r}}{I_{z} v_{x}} & 0 & \frac{-\left(C_{f}+C_{r}\right)}{I_{z} v_{x}}
\end{array}\right] \\
B_{v}=\left[\begin{array}{llll}
0 & \frac{C_{f}}{m} & 0 & \frac{a C_{f}}{I_{z}}
\end{array}\right]^{T} \\
C_{v}=\left[\begin{array}{llll}
0 & 0 & 0 & 1
\end{array}\right]
\end{gathered}
$$

where $C_{f}, C_{r}, a, b, m, I_{z}$ and $v_{x}$ are cornering stiffness of front and rear tires, distance from front and rear axle to the center of gravity of the vehicle, vehicle mass, yaw moment of inertia and the longitudinal velocity respectively. The vehicle transfer function is deduced from the state space model as follows:

$$
G_{v}=C_{v}\left(I s-A_{v}\right)^{-1} B_{v}
$$

\section{B. Steering actuator:}

The steering actuator is mainly represented by a steering column and an Electric Power System (EPS) that is regulated by a desired steering angle $\delta_{c}$ calculated by the controller. It is modeled by a $3^{\text {rd }}$ order transfer function $G_{a c t}$.

Thus, the general plant to be controlled is $G=G_{\nu} G_{a c t}$.

\section{Lateral controller:}

The design of $C_{1}$ (lane-changing controller) and $C_{2}$ (lanetracking controller) is based on $\mathrm{T} \& \mathrm{C}$ approach [4]. The controllers output is a steering wheel angle $\delta_{c}$ computed according to a yaw rate error $\omega_{r}=\omega_{v}-\omega_{d}$, the desired yaw rate is:

$$
\omega_{d}=v \rho_{\text {road }}
$$

where $\rho_{\text {road }}$ is the road curvature in the target point (set at a look-ahead distance $d$ ), and $v$ is the longitudinal velocity.

$$
\delta_{c}(s)=\frac{-k}{s}\left(\frac{d}{2 v}+\frac{1}{s}+\frac{v}{d s^{2}}\right) \omega_{r}(s)
$$

Thus,

$$
C(s)=\frac{-k}{s} \frac{\left(s^{2}+2 \frac{v}{d} s+2\left(\frac{v}{d^{2}}\right)\right)}{2 \frac{v}{d} s^{2}}
$$

$k$ and $d$ are designed in order to achieve the expected behaviors of $C_{1}$ and $C_{2}$, specifically :

- Lane-changing controller $C_{1}$ : In this case initial lateral error is significant. The controller must be smooth to avoid overshoots and uncomfortable sensation in the vehicle. For solving so, target point is set to $30 \mathrm{~m}$ (i.e. $d$ value).

- Lane-tracking controller $C_{2}$ : In this case, fast response is required. According to [4], the closer is the target point, the smaller is the tracking error. For doing so, look-ahead distance is fixed to $d=15 \mathrm{~m}$.

The appropriate feedback gain $k$ is identified using basic pole placement technique. Both controllers are implemented in a parametrized structure (see Fig. 3). As explained in section
II, the resultant Youla-Kucera controller switches between $C_{1}$ and $C_{2}$ without losing the stability of the closed loop. The parameter $\gamma$ is in charge of the controllers' switching based on the vehicle lateral error with respect to the trajectory as follows:

- When the lateral error $>3 m$, the adequate controller is $C_{1}$ so $\gamma=0$.

- When the lateral error $<0.2 m$, the adequate controller is $C_{2}$ so $\gamma=1$.

Between the two limits $\gamma$ changes gradually depending on the lateral error values as shown in Fig.4. Then $\gamma$ changes

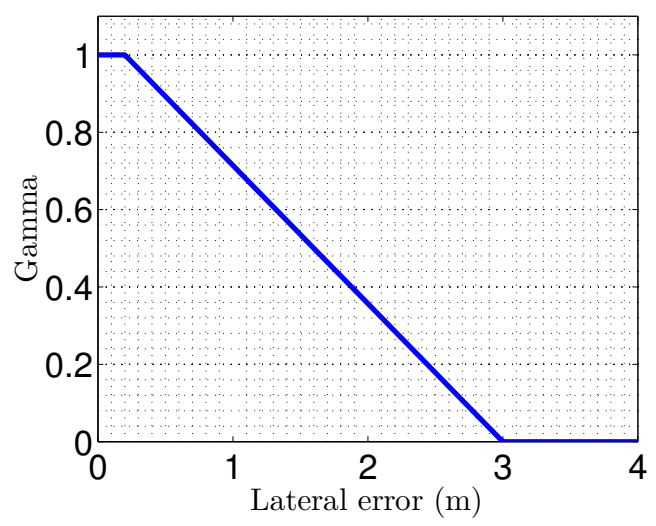

Fig. 4. The switching parameter $\gamma$

can be mathematically expressed by the equation below:

$$
\gamma=\left\{\begin{array}{cc}
0 & \mid \text { Lat }_{\text {error }} \mid>3 \\
-\frac{1}{2.8} \mid \text { Lat }_{\text {error }} \mid+\frac{3}{2.8} & 0.2<\mid \text { Lat }_{\text {error }} \mid<3 \\
1 & \mid \text { Lat }_{\text {error }} \mid<0.2
\end{array}\right.
$$

\section{Stability Analysis}

The closed loop stability of the full control structure is presented on this section. For doing so, the root locus response of the control structure for different $\gamma$ values is presented in Fig. 5.

It's notable in the root locus that all the poles during the transition are in the unity circle, which means that the whole system is stable. For $0<\gamma<1$, poles positions remain in $\left\{\right.$ poles $\left[G, C_{1}\right] \cup$ poles $\left.\left[G, C_{2}\right]\right\}$. Zeros appear and move to compensate $C_{1}$ or $C_{2}$ effects. The closer $\gamma$ to one, the closer the zeros to poles of $\left[G, C_{1}\right]$, and respectively the closer $\gamma$ to zero the closer the zeros to poles of $\left[G, C_{2}\right]$. In Fig.5 a part of the root locus is zoomed, zeros and poles are represented respectively by circles and crosses. It is shown that zeros move between $\left[G, C_{1}\right]$ 's poles and $\left[G, C_{2}\right]$ 's poles with respect to $\gamma$.

\section{SIMULATION RESULTS}

To validate the algorithm, a given scenario was implemented using the vehicle model previously described (see section III for details). A straight lane is used as reference trajectory. All simulations are started with an initial deviation of 3 meters from the reference trajectory and the vehicle speed was set to $10 \mathrm{~m} / \mathrm{s}$. 


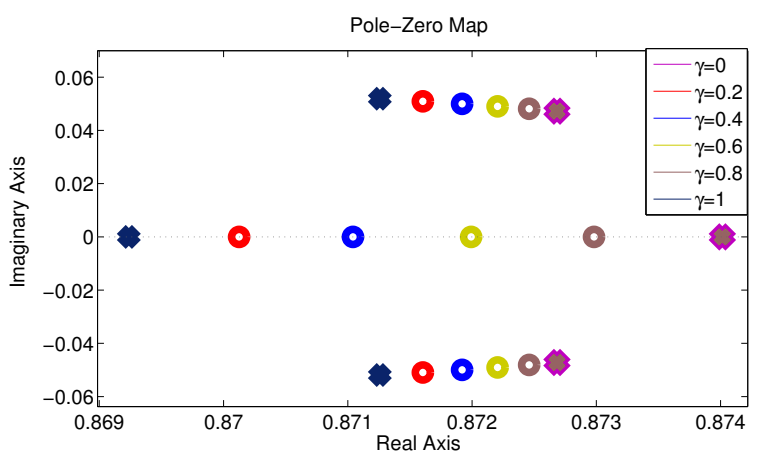

Fig. 5. Dynamics compensation

Figure 6 shows the performance of the controller $\mathrm{C} 1$ designed for lane-tracking response (solid black line); the controller $\mathrm{C} 2$ designed for lane-change situations (solid blue line) and the proposed YK. one (solid red line) with respect to the reference trajectory (solid green line).

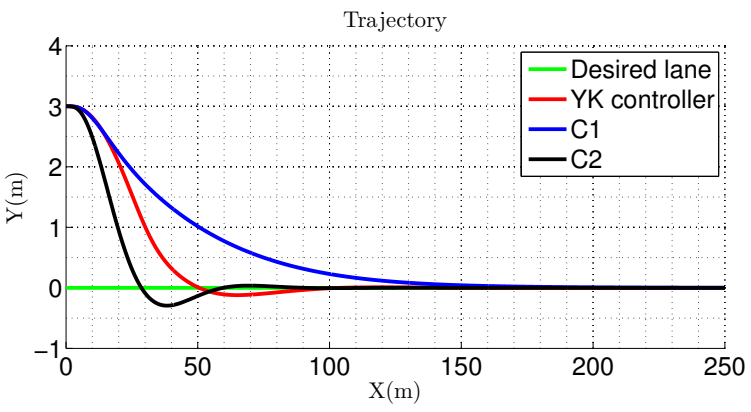

Fig. 6. Response for constant speed $v=10 \mathrm{~m} / \mathrm{s}$

The vehicle controlled by $C_{2}$ (lane-tracker controller) is the first to reach the desired lane, but Figure 7 shows that its steering wheel angle is the highest with a brutal steering rate, that causes uncomfortable sensation in the vehicle, the vehicle controlled by $C_{1}$ (lane-change controller) has the smoothest steering angle and the lowest steering rate but it takes around $150 \mathrm{~m}$ to reach the lane, while the vehicle controlled by the proposed approach (YK controller) has a correct behavior, it reaches the desired lane at the same time that the one controlled by $C_{2}$ while maintaining smoothness of steering angle and steering rate, it satisfies the required steering performance .

\section{EXPERIMENTAL VALIDATION}

The proposed approach was tested on an electric Renault ZOE that has been modified for allowing steering computed control.

To validate the proposed control structure, many scenarios have been tested. In each test the vehicle was removed manually from the desired lane and the autonomous mode was started at a given lateral error as shown in Fig.8. The manual driven phases are denoted by $\mathbf{M}$. The vehicle speed is set to $10 \mathrm{~m} / \mathrm{s}$.

In Figure 8 it's shown that the controller switched between
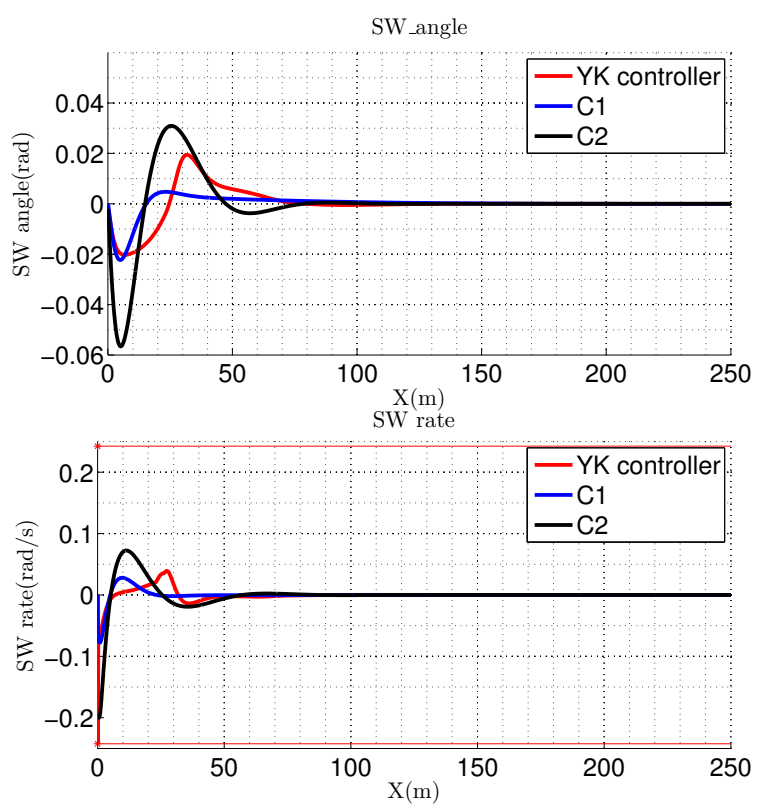

Fig. 7. Steering wheel angle and steering wheel rate

$C_{1}$ and $C_{2}$ gradually according to the lateral error value (see $\gamma)$. The vehicle was able to reach the desired lane in less than 10 seconds in all cases. It performed smooth behavior even for 5m lateral deviation. In Fig. 9 two scenarios are shown. The overshots remained less than $0.1 \mathrm{~m}$. It's also worth to mention that in each test the vehicle maintained comfortable steering as in manual driving.
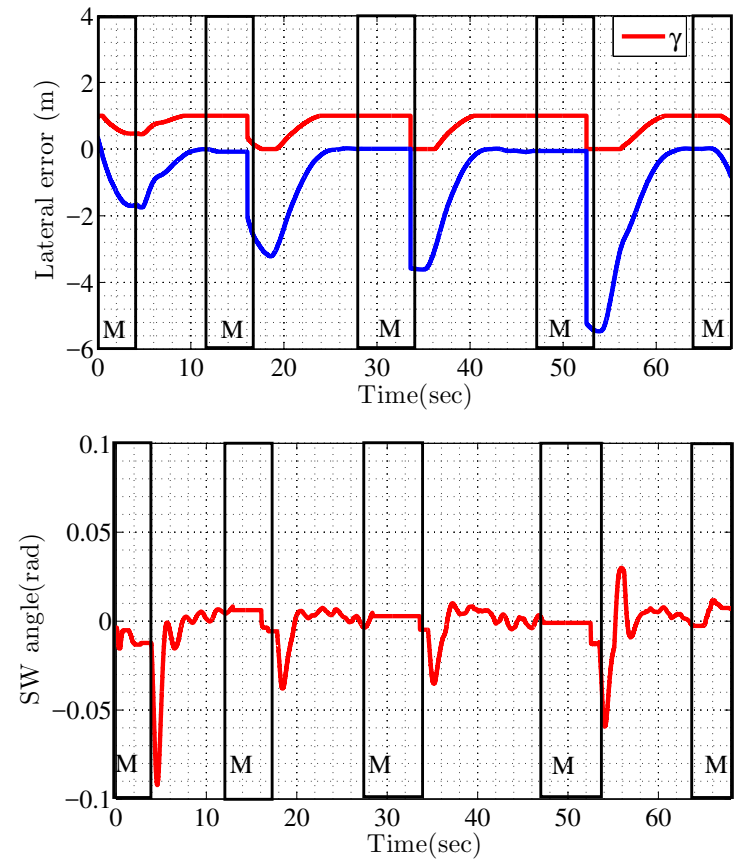

Fig. 8. Experimental results 

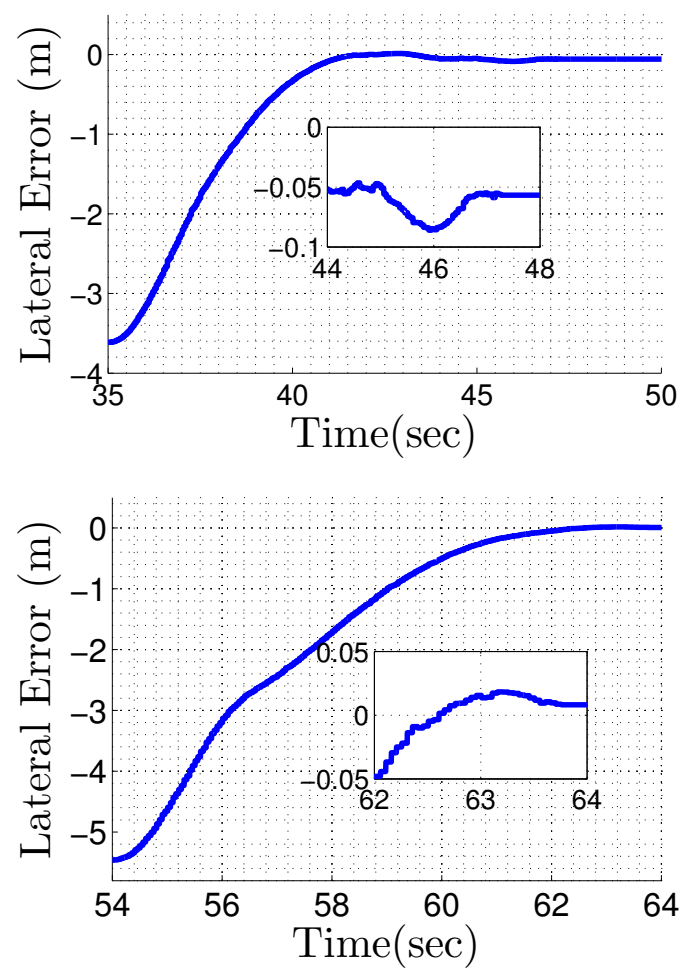

Fig. 9. Lateral error

\section{CONCLUSION}

Autonomous vehicles should be able to address and respond to any circumstance, meaning that the steering system should be able to perform different maneuvers (i.e. lane changing, lane tracking, avoiding objects), while maintaining stability, control performance and passengers comfort. This paper proposed a Youla-Kucera based control structure including two controllers: one for tracking the desired trajectory and one for lane changing case.

Experimental results have proven that the proposed control structure is a good way of dealing with this situations. It enhances performances of only using a single controller for two cases. Errors are significantly reduced, and even with a deviation of $5 \mathrm{~m}$ the vehicle reached the desired lane smoothly. The switching between controllers was gradual and stable.

Further works will be oriented to include more driving situations to enlarge controller capabilities and ensure high performance in any circumstance without loosing steering system stability or compromising passengers comfort. Finally converging to a system able to control the vehicle at full range speeds.

\section{ACKNOWLEDGMENT}

Authors express their gratitude to the RENAULT research department for its support in developing experimental tests. This paper reflects solely the views of the authors and not necessarily the views of the company they belong to.

\section{REFERENCES}

[1] Tay, T. T., Mareels, I., \& Moore, J. B. (2012). High performance control. Springer Science \& Business Media.

[2] Niemann, H., \& Stoustrup, J. (1999). An architecture for implementation of multivariable controllers. In American Control Conference, 1999. Proceedings of the 1999 (Vol. 6, pp. 4029-4033). IEEE.

[3] Stoustrup, J. (2009). Plug \& play control: Control technology towards new challenges. European Journal of Control, 15(3-4), 311-330..

[4] Tan, H. S., \& Huang, J. (2014). Design of a high-performance automatic steering controller for bus revenue service based on how drivers steer. IEEE Transactions on Robotics, 30(5), 1137-1147.

[5] Luan, B. C., Lee, I. H., Tan, H. S., Li, K., Yuan, D., \& Chou, F. C. (2016). Design and Field Testing of a Lane Following Control System with a Camera Based on T\&C Driver Model (No. 2016-01-0117). SAE Technical Paper.

[6] Jochem, T., \& Pomerleau, D. (1996). Life in the fast lane: The evolution of an adaptive vehicle control system. AI magazine, 17(2), 11.

[7] Broggi, A., Bertozzi, M., Fascioli, A., Bianco, C. G. L., \& Piazzi, A. (1999). The ARGO autonomous vehicles vision and control systems. International Journal of Intelligent Control and Systems, 3(4), 409441.

[8] Thrun, S., Montemerlo, M., Dahlkamp, H., Stavens, D., Aron, A., Diebel, J., ...\& Lau, K. (2006). Stanley: The robot that won the DARPA Grand Challenge. Journal of field Robotics, 23(9), 661-692.

[9] Broggi, A., Medici, P., Zani, P., Coati, A., \& Panciroli, M. (2012). Autonomous vehicles control in the VisLab intercontinental autonomous challenge. Annual Reviews in Control, 36(1), 161-171.

[10] Jo, K., Lee, M., Kim, D., Kim, J., Jang, C., Kim, E., ... \& Huh, K. (2013). Overall reviews of autonomous vehicle a1-system architecture and algorithms. IFAC Proceedings Volumes, 46(10), 114-119.

[11] Ziegler, J., Bender, P., Schreiber, M., Lategahn, H., Strauss, T., Stiller, C., ...\& Kaus, E. (2014). Making Bertha driveAn autonomous journey on a historic route. IEEE Intelligent Transportation Systems Magazine, 6(2), 8-20.

[12] Marino, R., Scalzi, S., \& Netto, M. (2011). Nested PID steering control for lane keeping in autonomous vehicles. Control Engineering Practice, 19(12), 1459-1467.

[13] Hima, S., Lusseti, B., Vanholme, B., Glaser, S., \& Mammar, S. (2011). Trajectory tracking for highly automated passenger vehicles. IFAC Proceedings Volumes, 44(1), 12958-12963.

[14] Tagne, G., Talj, R., \& Charara, A. (2013, June). Higher-order sliding mode control for lateral dynamics of autonomous vehicles, with experimental validation. In Intelligent Vehicles Symposium (IV), 2013 IEEE (pp. 678-683). IEEE.

[15] Onieva, E., Naranjo, J. E., Milanés, V., Alonso, J., Garcìa, R., \& Pérez, J. (2011). Automatic lateral control for unmanned vehicles via genetic algorithms. Applied Soft Computing, 11(1), 1303-1309.

[16] Niemann, H. (2006). Parameterisation of extended systems. IEE Proceedings-Control Theory and Applications, 153(2), 221-227.

[17] Kucera, V. (1975). Stability of discrete linear feedback systems. IFAC Proceedings Volumes, 8(1), 573-578.

[18] Borrelli, F., Falcone, P., Keviczky, T., Asgari, J.,\& Hrovat, D. (2005). MPC-based approach to active steering for autonomous vehicle systems. International Journal of Vehicle Autonomous Systems, 3(2-4), 265-291.

[19] Rajamani, R. (2011). Vehicle dynamics and control. Springer Science $\&$ Business Media. 